\title{
An Ultra-Thin 2-bit Near-Field Transmitarray Lens
}

\author{
Enrique G. Plaza ${ }^{1}$, G. León ${ }^{1}$, S. Loredo ${ }^{1}$, A. Arboleya ${ }^{1}$, F. Las-Heras ${ }^{1}$, C.M. Suárez ${ }^{2}$, M. R. Pino ${ }^{1}$, \\ ${ }^{1}$ Area of Signal Theory and Communications, Universidad de Oviedo, Gijón, Spain, \\ \{ egplaza, gleon, sloredo, aarboleya, flasheras, mpino \}@tsc.uniovi.es \\ ${ }^{2}$ Area of Manufacturing Processes, Universidad de Oviedo, Gijón, Spain.
}

\begin{abstract}
A novel ultra-thin Near-Field 2-bit 24x24-element transmitarray lens working at $\mathrm{X}$-Band is presented in this letter. The aim of this antenna is to focus in a point located in the Near-Field without using any complex feeding network. In order to obtain a planar lens with a very low profile, a 2-bit-design approach is used to diminish the number of layers used in the structure. The simulations have been done using a model based on the concept that the Near-Field of an array can be calculated as the sum of the Far-Field contributions of every single one of its elements. These are considered as plane apertures over which the tangential electric field is constant. In order to validate the results of this model, they are compared with both those obtained with a full-wave commercial software and those from measurements. Thus, a Near-Field-antenna is manufactured and measured showing the measurements good concordance with the results of the model.
\end{abstract}

Keywords - Focusing, transmitarray, quasi-periodic surface, Near-Field

\section{INTRODUCTION}

The recent development of short-range applications has increased the relevance of Near-Field (NF) antennas. Therefore, designs for RFID applications [1],[2], medical diagnosis [3] or any other general-purpose application involving detecting near objects can be found in the literature. Most of these antennas are based on phased arrays [4], which normally present a complex feeding network, or Fresnel lenses [5], which present low efficiency. Thus, a planar lens antenna could be used to overcome these problems, since it is no longer necessary to make use of a feeding network and the antenna can be designed to maximize the transmitted power.

Therefore, the overall importance of antennas based on planar lenses has significantly grown [6]-[8], with the transmitarray (TA) being one of the most important examples. Nonetheless, in this type of antennas, in order to obtain the necessary phase-shift, it is often required to use a 4-layer structure [9]. This is due to the necessity of obtaining a $360^{\circ}$ phase-shift while the variation of the transmitted amplitude is kept below $-3 \mathrm{~dB}$, by changing one or more of the unit cell elements. This may sometimes result into structures that increase the weight of the system. One of the solutions is to quantify the phase-shift that each cell should introduce. For instance, in the case of a 1-bit structure it would only require a phase delay of $0^{\circ}$ or $180^{\circ}$ [10]. This means that the maximum phase error introduced by any unit cell is $90^{\circ}$. Nevertheless, this feature could be lowered by using a higher number of bits, although the most common quantifications are the ones using 1 and 2 bits [11], because a 2-layer structure can be used.

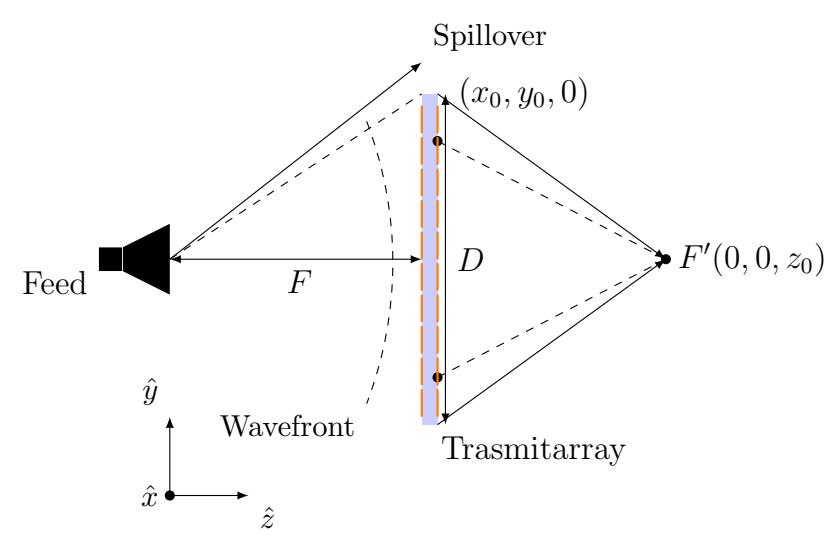

Fig. 1. Scheme of the complete structure.

Thus, a novel NF antenna based on a TA is presented in this paper. A scheme of the system is depicted in Fig. 1, being $\mathrm{F}$ the focal distance and D the size of the lens. The antenna is fed by a horn located in its focal point and the unit cells are modified to introduce the necessary phase-shift in order to focus at a certain point located in the NF $\left(F^{\prime}\right)$.

\section{UNIT CELL}

The design of the 2-bit unit cell of this TA, at $10 \mathrm{GHz}$, is based on a variation of a 4-dielectric-layer structure made of 4 square stacked patches coupled 2 by 2 by slot [12], which yields a stable behavior when the incident angle is varied, whereas a phase-shift of $360^{\circ}$ can be obtained. Then, by introducing a 2-bit phase-quantification, the number of dielectric layers forming the stacked structure can be reduced to only 2, as shown in Fig. 2. In this design, the slot has been turned into a symmetric cross in order to obtain dual-polarization. In Table I, an overview of the results of the final design is shown where $\tau$ is the coefficient transmission of the element. From this point on, only one of the polarizations will be taken into account for the analysis since the structure is symmetric and the results of both polarizations are the same. Hence, it can be observed that, by using a 2-bit unit cell, it is possible to obtain the necessary phase-shift for this structure while maintaining the level of the transmitted amplitude. Although the last of the quantification levels is placed in the limits of usage, it does not significantly affect the behavior of the structure. These simulations were carried out using the full-wave commercial software Ansys HFSS. 


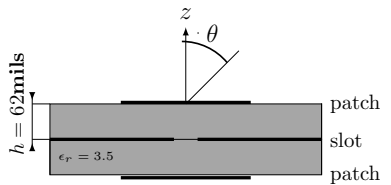

(a)

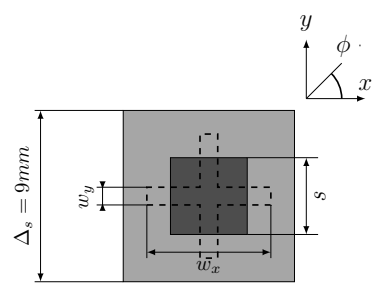

(b)
Fig. 2. Scheme of the unit cell. (a) Profile view. (b) Top view.

TABLE I

UNIT CELL RESULTS AT @10 GHz

\begin{tabular}{cccccc}
\hline Bit & $w_{x}(\mathrm{~mm})$ & $w_{y}(\mathrm{~mm})$ & $S(\mathrm{~mm})$ & $|\tau|(\mathrm{dB})$ & $\angle \tau(\mathrm{deg})$. \\
\hline 00 & 6.875 & 0.75 & 1.5 & -3.00 & 0.0 \\
01 & 6.875 & 0.75 & 3.9 & -2.14 & -90.57 \\
10 & 6.875 & 0.75 & 6.3 & -0.37 & -180.75 \\
11 & 7.5 & 0.75 & 6.8 & -3.34 & -222.19 \\
\hline
\end{tabular}

\section{MODEL}

In order to carry out a rapid and efficient simulation of the NF of a TA, a model similar to the ones in [13]-[15] is presented in this paper. In this model, the final NF of the structure is calculated as the sum of the FF contributions of all its elements. It is assumed that all these elements are square apertures over which the tangential electric field $\left(E_{t a n}^{x}\right)$ is constant. The FF contribution $\left(E_{x ; m, n}^{X}\right)$ of the element in the $(m, n)$ position of the mesh is calculated using the equations (1) to (6). Then, all these contributions are summed to obtain the total electric field (7). In these equations, $\phi$ and $\theta$ are the spherical coordinates given that the origin of each FF contribution is located in the center of each element, $k$ is the wave number and $r$ is the distance from the center of each cell to the point where the field is calculated. In this case, the field is acquired in a planar regular meshed aperture of $M x N$ elements located at the plane of the lens. The value of $E_{t a n}^{x}$ in the lens zone (shaded area in Fig. 3) is given by the product between the incident field and the coefficient transmission of each unit cell and in the spillover area (dotted zone area in Fig. 3) by the field of the horn antenna.

$$
\begin{gathered}
\Psi=\frac{k \Delta_{s} \sin \theta \cos \phi}{2 \pi} \\
\Phi=\frac{k \Delta_{s} \sin \theta \sin \phi}{2 \pi} \\
k=\frac{2 \pi}{\lambda} \\
E_{\theta}^{x}=\frac{j k e^{-j k r} \Delta_{s}^{2} E_{t a n}^{x} \cos (\phi)}{2 \pi r} \operatorname{sinc}(\Psi) \operatorname{sinc}(\Phi) \\
E_{\phi}^{x}=\frac{-j k e^{-j k r} \Delta_{s}^{2} E_{t a n}^{x} \sin (\phi) \cos (\theta)}{2 \pi r} \operatorname{sinc}(\Psi) \operatorname{sinc}(\Phi) \\
E_{x ; m, n}^{X}=\cos \theta \cos \phi E_{\theta}^{x}-\sin \phi E_{\phi}^{x}
\end{gathered}
$$

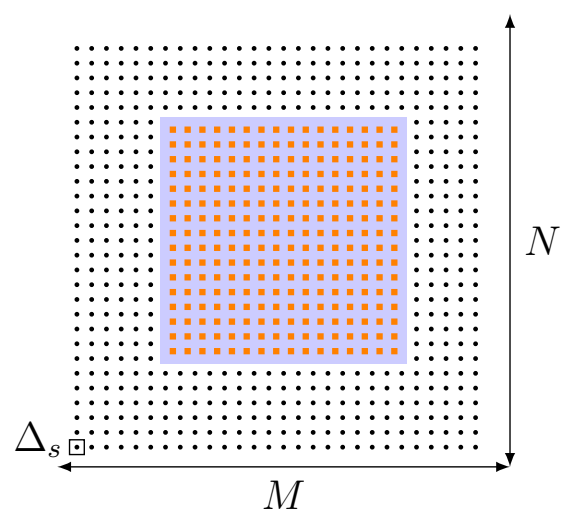

Fig. 3. Scheme of the field aperture for calculating the NF.

$$
E_{x, T O T}^{X}=\sum_{n=1}^{N} \sum_{m=1}^{M} E_{x ; m, n}^{X}
$$

This model is capable of analyzing a NF system in approximately 15 minutes in a desktop computer, whereas it can take one day to carry out the same simulation using a full-wave software like HFSS v15 in a workstation (Ax AMD Opteron 8378 with 128 GB of RAM memory).

\section{TRANSMITARRAY DESIGN}

In this letter, a 2-bit TA has been designed for a NF application in the $\mathrm{X}$-Band at $10 \mathrm{GHz}$, in which the feed is a corrugated horn with a directivity of $11 \mathrm{dBi}$. The characteristic parameters of this antenna are: $F^{\prime}=F=151.2 \mathrm{~mm}$ and $D=216 \mathrm{~mm}$, equivalent to 24 unit cells per side, see shaded zone in Fig. 3. In addition, the values of $N$ and $M$ will be set to 224 for the rest of the analysis. This TA is designed with a process similar to the one used for a NF phased array. It is intended to focus at a certain distance from the antenna at a point placed in the axis perpendicular to the system (Z-Axis), and each element of the array introduces the necessary phase-shift. Nonetheless, in this case, the different cells are chosen in order to minimize the difference between the ideal phase that each cell must introduce $\left(\Omega_{r e q}\right)$ and the different levels of the quantified phase-shift. In Fig. 4 the comparison between the reconstructed phase for the ideal and the 2-bit case is depicted, showing that, while there is a certain quantification error in the reconstructed phase, it is not significant. This error $\left(\Omega_{\text {error }}\right)$ would be calculated as the modulus of the difference between the phase reconstructed by each cell $\left(\Omega_{\text {cell }}\right)$ and the one required for the ideal case $\left(\Omega_{r e q}\right)$ according to (8), in which $x_{i}$ and $y_{i}$ are the coordinates of the cell center and $\angle E_{i n c}$ the phase of the impinging field:

$$
\begin{gathered}
\Omega_{r e q}=k\left(\sqrt{x_{i}^{2}+y_{i}^{2}+\left(F^{\prime}\right)^{2}}-F^{\prime}\right)-\angle E_{i} \\
\Omega_{\text {error }}=\left|\Omega_{\text {cell }}-\Omega_{\text {req }}\right|
\end{gathered}
$$




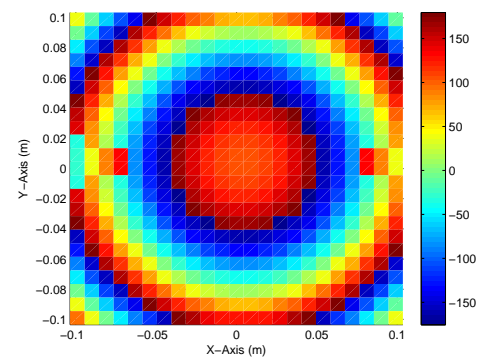

(a)

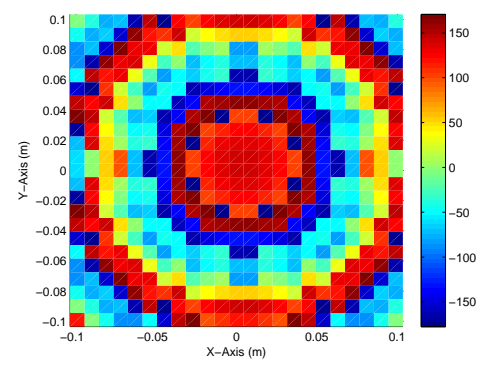

(b)

Fig. 4. Reconstructed phase distribution. (a) Ideal case. (b) 2-bit case.

\section{Measurements}

This 2-bit TA was manufactured and measured in a planar range [16] in order to validate the model described in Section III. Fig. 5 shows a picture of the fabricated prototype where the different size of the patches for the different cells can be appreciated.

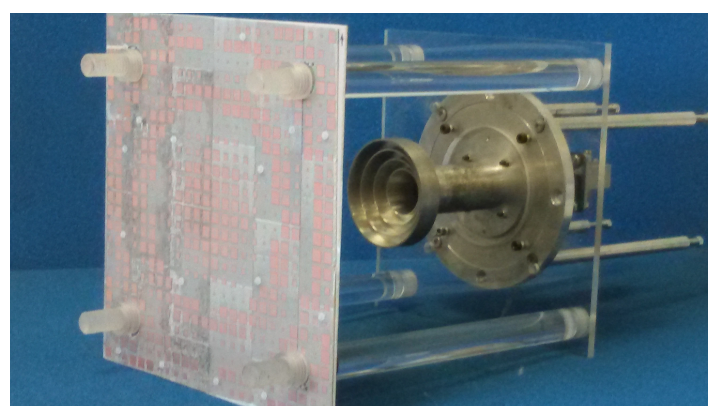

Fig. 5. Manufactured prototype.

Fig. 6 compares the different results in the Z-Axis. For a better comparison, these are normalized to the maximum of the field obtained with the lens. Thus, it can be observed that there is good concordance between the model, the full-wave simulation and the measurement data, since the maxima are located very close to each other and the curves present the same $-3 \mathrm{~dB}$ beamwidth. The small variations that can be seen in the measurement are due to the reflection and diffraction among the different supporting elements used to carry out the process.

Figs. 7(a) and 7(b) show the comparison between the model results and measurements for the principal cuts of the plane located at the point where the field of the antenna has its maximum $(z=135 \mathrm{~mm})$, which is slightly closer to the lens

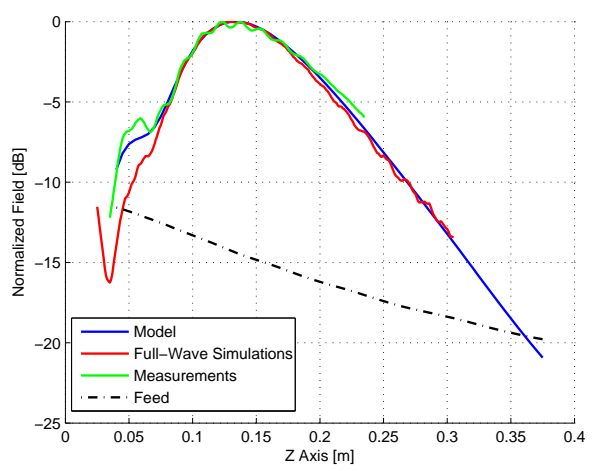

Fig. 6. Comparison of model and measurements for the Z-Axis at $10 \mathrm{GHz}$.

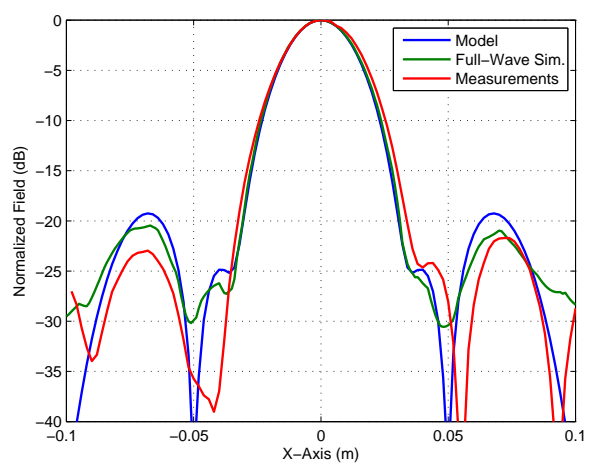

(a) X-Axis

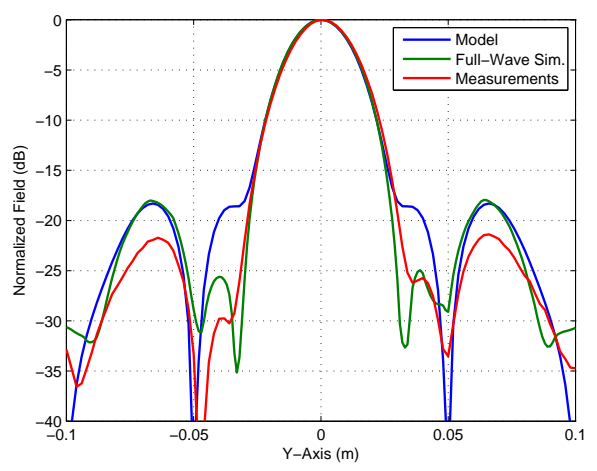

(b) Y-Axis

Fig. 7. Comparison between measurements and simulations at $10 \mathrm{GHz} . z=$ $135 \mathrm{~mm}$. (a) X-Axis. (b) Y-Axis.

than $F^{\prime}$. In this case, the location of the maximum of the field depends on the size of the lens. It can be seen that there is also good agreement between the model and the experimental results. In Table II, the data obtained with the model and measurements concerning the Spotwidth $(\mathrm{Sw})$,which is the width in $\mathrm{mm}$. of the beam measured at 1 or $3 \mathrm{~dB}$ below the maximum, and the level of secondary lobes are compared, showing very similar values.

Finally, a characterization of the variation of the system behavior with the frequency is carried out in order to estimate the bandwidth of the antenna. The following study has been carried out in terms of the focusing gain (FG) of the system, 
TABLE II

Simulations \& MEASUREMENTS RESUlts

\begin{tabular}{cccc}
\hline Axis & $-1 \mathrm{~dB} \mathrm{Sw}$ & $-3 \mathrm{~dB} \mathrm{Sw}$ & $S L L(\mathrm{~dB})$ \\
\hline X meas. & $17 \mathrm{~mm}$ & $28 \mathrm{~mm}$ & -22.9 \\
X sim. & $16 \mathrm{~mm}$ & $26 \mathrm{~mm}$ & -19.0 \\
Y meas. & $14 \mathrm{~mm}$ & $25 \mathrm{~mm}$ & -18.3 \\
Y sim. & $14 \mathrm{~mm}$ & $25 \mathrm{~mm}$ & -21.7 \\
\hline
\end{tabular}

which is defined as the ratio between the field obtained with the lens $\left(E_{T A}^{x}\right)$ and the one obtained without it $\left(E_{F E E D}^{x}\right)$ at each point of the space:

$$
F G(r, \theta, \phi)=\frac{E_{T A}^{x}(r, \theta, \phi)}{E_{F E E D}^{x}(r, \theta, \phi)}
$$

Fig. 8 depicts the results of this parameter at the point where the maximum of the field at $10 \mathrm{GHz}$ is placed, i.e., $z=135 \mathrm{~mm}$, showing good concordance between the model that has been used to characterized the system and the measurements. In addition, the bandwidth predict by the model is slightly wider than the one obtained in simulation. Nonetheless, this feature is around the $20 \%$ of the central frequency, which is a good indicator for this type of antenna, and, therefore, the difference of bandwidth does not have a significant effect. Besides, the results corresponding to the simulation in HFSS of an equivalent Fresnel lens in terms of aperture and feeding antenna are depicted. Thus, it can bee seen that the performance of the prototype improves the behavior of the latter.

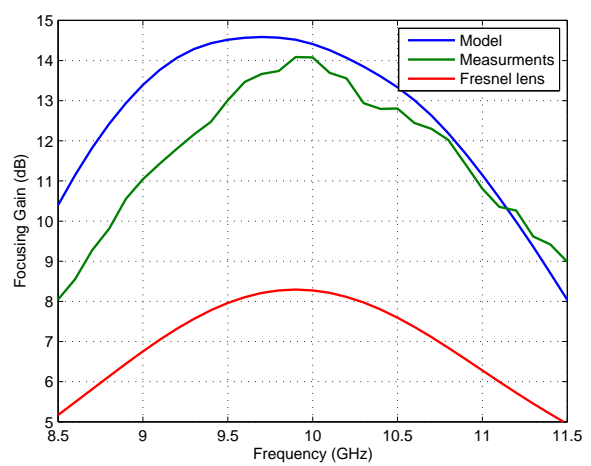

Fig. 8. Focusing gain of the system vs. frequency for $z=135 \mathrm{~mm}$.

\section{CONCLUSIONS}

In this letter, an ultra-thin NF-TA based on a 2-bit unit cell formed by 2 square stacked patches coupled by a symmetric cross is presented. The 2-bit TA was designed, manufactured and measured in order to validate the results obtained in simulations. These were carried out by using a model that performs a fast calculation of the NF by summing the FF contributions of all the elements of the system. In this sense, a good concordance has been obtained between simulations and measurements in both the $\mathrm{Z}$ - and the $\mathrm{X}$ - and Y-Axis, validating the results given by the model. Besides, a $25-\mathrm{mm}$ quasi-circular spot has been obtained while the value of the secondary lobes is lower than $-15 \mathrm{~dB}$, which is a good performance for a NF-antenna of this type. To sum up, the results obtained in this study show that it is possible to obtain a good behavior while reducing the overall size of the TA.

\section{ACKNOWLEDGMENT}

The present work has been developed under the support of the FPI pre-doctoral grant with ref no. BES-2012-053154, the project MIRIIEM with ref. TEC2014-540005-P of the Ministerio de Economía y Competitividad, and the research project of the Gobierno del Principado de Asturias / FEDER with ref. GRUPIN14-114.

\section{REFERENCES}

[1] X. Qing, C.K. Goh, and Z.N. Chen, Segmented loop antenna for UHF near-field RFID applications, Electronics Letters, vol. 45, no. 17, pp. 872 - 873, Aug. 2009.

[2] A. Michel and P. Nepa, UHF-RFID desktop reader antennas: performance analysis in the near-field region, IEEE Antennas and Wireless Propagation Letters, vol. 15, pp. 1430 - 1433, 2016.

[3] F. T. J. Nourinia, Near-field focused array microstrip planar antenna for Medical Applications, IEEE Antennas and Wireless Propagation Letters, vol. 13, pp. 951-954, 2014

[4] J. Álvarez, R. G. Ayestarán, G. León, L. F. Herrán, A. Arboleya, J. A. López-Fernndez, and F. Las-Heras, Near field multifocusing on antenna arrays via non-convex optimisation, IET Microw. Antennas Propag., Vol. 8, Iss. 10 , pp. 754-764, Jul. 2014

[5] S. Karimkashi and A. A. Kishk, Focusing properties of Fresnel zone plate lens antennas in the near-field region, IEEE Transactions on Antennas and Propagation, vol. 59, no. 5, pp. 1481-1487, June 2011.

[6] N. Gagnon, A. Petosa, and D.A. McNamara, Research and development on phase-shifting surfaces (PSSs), IEEE Antennas and Propagation Magazine, vol. 55, no. 2, pp. 29-48, Apr. 2013

[7] C.G. Ryan, M.R. Chararmir, J. Shaker, J.R. Bray, Y.M.M. Antar, and A Ittipiboon, Wideband transmitarray using dual-resonant double square rings, IEEE Transactions on Antennas and Propagation, vol. 58, no. 5, pp. 1486-1493, May 2010.

[8] A.H. Abdelrahman, A.Z. Elsherbeni, and F. Yang, Transmitarray antenna design using cross-slot elements with no dielectric substrate, IEEE Antennas and Wireless Propagation Letters, vol. 13, pp. 177-180, 2014.

[9] H. Nematollahi, J.-J. Laurin, J. E. Page and J. A. Encinar, Design of broadband transmitarray unit cells with comparative study of different numbers of layers, IEEE Transactions on Antennas and Propagation, vol. 63, no. 4, pp. 1473-1481, May. 2015.

[10] W. Pan, C. Huang, X. Ma, B. Jiang and X. Luo, A dual linearly polarized transmitarray element with 1 -Bit phase resolution in X-band, IEEE Antennas and Wireless Propagation Letters, vol.14, pp. 167-170, 2015.

[11] W. Pan, C. Huang, X. Ma, B. Jiang, and X. Luo, Design and demonstration of 1-bit and 2-bit transmit-arrays at $X$-band frequencies, Proceedings of the 39th European Microwave Conference, pp. 918-921, Oct. 2009.

[12] E. G. Plaza, S. Loredo, G. León, and F. Las-Heras, Comparison of different structures for transmitarrays cells, Microwave and Optical Technology Letters, vol. 56, no. 6, pp. 1295-1299, Jun. 2013.

[13] E. G. Plaza, G. León, S. Loredo, and F. Las-Heras, Near-field focusing transmitarray lens, 9th European Conference on Antennas and Propagation (EuCAP), Lisbon, Apr. 2015.

[14] A. Buffi, A. A. Serra, P. Nepa, H-T. Chou, and G. Manara, A Focused Planar Microstrip Array for $2.4 \mathrm{GHz}$ RFID Readers, IEEE Transactions on Antennas and Propagation, vol. 58, no.5, pp. 1536-1544, May 2010.

[15] D. R. Prado, A. F. Vaquero, M. Arrebola, M. R. Pino, and F. Las-Heras, Optimization of the reflectarray quiet zone for use in compact antenna test range, AMTA Symposium, Long Beach CA., 2015

[16] A. Arboleya, Y. Álvarez and F. Las-Heras, Millimeter and sub-millimeter planar measurement setup , 2013 IEEE Antennas and Propagation Society International Symposium, Orlando(FL), 2013. 